\title{
The Truth About HTML5
}

\section{Luke Stevens}

RJ Owen 


\section{The Truth About HTML5}

\section{Copyright ( 2014 by Luke Stevens and RJ Owen}

This work is subject to copyright. All rights are reserved by the Publisher, whether the whole or part of the material is concerned, specifically the rights of translation, reprinting, reuse of illustrations, recitation, broadcasting, reproduction on microfilms or in any other physical way, and transmission or information storage and retrieval, electronic adaptation, computer software, or by similar or dissimilar methodology now known or hereafter developed. Exempted from this legal reservation are brief excerpts in connection with reviews or scholarly analysis or material supplied specifically for the purpose of being entered and executed on a computer system, for exclusive use by the purchaser of the work. Duplication of this publication or parts thereof is permitted only under the provisions of the Copyright Law of the Publisher's location, in its current version, and permission for use must always be obtained from Springer. Permissions for use may be obtained through RightsLink at the Copyright Clearance Center. Violations are liable to prosecution under the respective Copyright Law.

ISBN-13 (pbk): 978-1-4302-6415-6

ISBN-13 (electronic): 978-1-4302-6416-3

Trademarked names, logos, and images may appear in this book. Rather than use a trademark symbol with every occurrence of a trademarked name, logo, or image we use the names, logos, and images only in an editorial fashion and to the benefit of the trademark owner, with no intention of infringement of the trademark.

The use in this publication of trade names, trademarks, service marks, and similar terms, even if they are not identified as such, is not to be taken as an expression of opinion as to whether or not they are subject to proprietary rights.

While the advice and information in this book are believed to be true and accurate at the date of publication, neither the authors nor the editors nor the publisher can accept any legal responsibility for any errors or omissions that may be made. The publisher makes no warranty, express or implied, with respect to the material contained herein.

President and Publisher: Paul Manning

Lead Editor: Ben Renow-Clarke

Technical Reviewer: Andrew Zack

Editorial Board: Steve Anglin, Mark Beckner, Ewan Buckingham, Gary Cornell, Louise Corrigan, Jim DeWolf, Jonathan Gennick, Jonathan Hassell, Robert Hutchinson, Michelle Lowman, James Markham, Matthew Moodie, Jeff Olson, Jeffrey Pepper, Douglas Pundick, Ben Renow-Clarke, Dominic Shakeshaft, Gwenan Spearing, Matt Wade, Steve Weiss

Coordinating Editor: Kevin Shea

Copy Editor: Kim Wimpsett

Compositor: SPi Global

Indexer: SPi Global

Artist: SPi Global

Cover Designer: Anna Ishchenko

Distributed to the book trade worldwide by Springer Science+Business Media New York, 233 Spring Street, 6th Floor, New York, NY 10013. Phone 1-800-SPRINGER, fax (201) 348-4505, e-mail orders-ny@springer-sbm.com, or visit WwW.springeronline.com. Apress Media, LLC is a California LLC and the sole member (owner) is Springer Science + Business Media Finance Inc (SSBM Finance Inc). SSBM Finance Inc is a Delaware corporation.

For information on translations, please e-mail rights@apress.com, or visit www.apress.com.

Apress and friends of ED books may be purchased in bulk for academic, corporate, or promotional use. eBook versions and licenses are also available for most titles. For more information, reference our Special Bulk Sales-eBook Licensing web page at WWW. apress.com/bulk-sales.

Any source code or other supplementary material referenced by the author in this text is available to readers at WWW. apress . com. For detailed information about how to locate your book's source code, go to WWw. apress.com/source-code/. 


\section{Contents at a Glance}

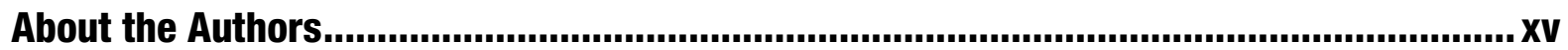

About the Technical Reviewer ................................................................................. xvii

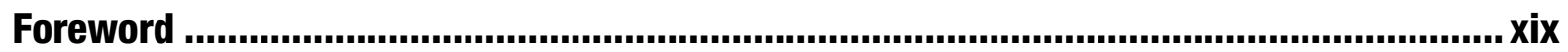

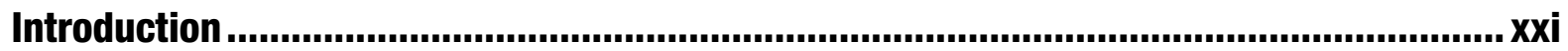

Chapter 1: A Somewhat Sensationalized History of HTML5 ......................................... 1

Chapter 2: The Truth About a Basic HTML5 Web Page...................................................13

Chapter 3: The Truth About Structuring an HTML5 Page ............................................17

Chapter 4: The Truth About HTML5's Structural Elements..............................................31

Chapter 5: The Truth About HTML5 Micro-semantics and Schema.org ..........................47

Chapter 6: The Truth About HTML5 and SEO............................................................59

Chapter 7: The Truth About HTML5's Other New Elements ...........................................61

Chapter 8: The Truth About HTML5 Forms................................................................67

Chapter 9: The Truth About HTML5's Canvas, Gaming, and Flash...................................81

Chapter 10: The Truth About Audio and Video in HTML5 ..............................................117

Chapter 11: The Truth About SVG: The Flash Challenger That Was,

Wasn't, and Now.... ...............................................................................135

Chapter 12: The Truth About HTML5 Web Apps, Mobile, and What Comes Next............153

Chapter 13: The Truth About the Future of Web Design: Performance-Based Design..... 165

Index. 



\section{Contents}

About the Authors...................................................................................................... XV

About the Technical Reviewer ..................................................................................... xvii

Foreword ................................................................................................................ xix

Introduction ................................................................................................................ Xxi

Chapter 1: A Somewhat Sensationalized History of HTML5 ........................................ 1

How Architecture Astronauts and the W3C Tried to Kill HTML ................................................1

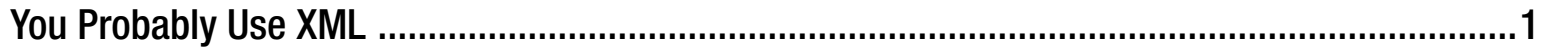

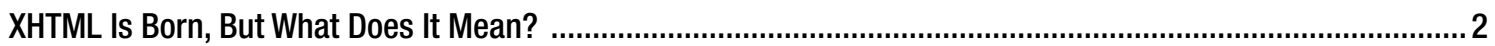

Draconian Error Handling (Or: Why Don't I Just Punch You in the Face?) ….....................................................2

OK, Not Really. But Your Browser Would Punch You in the Face .................................................................... 3

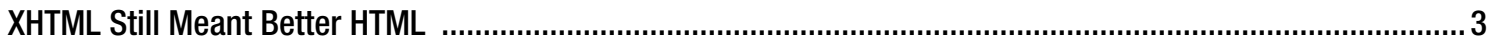

But the Crazy Had Only Just Begun ..................................................................................................

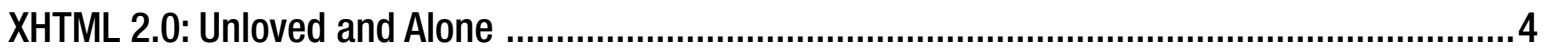

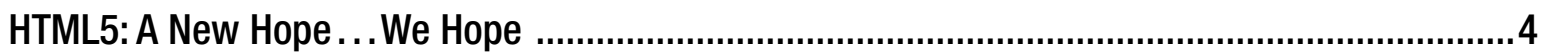

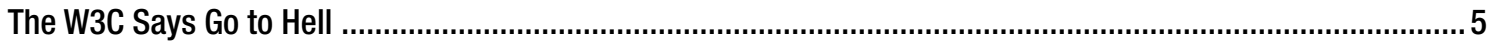

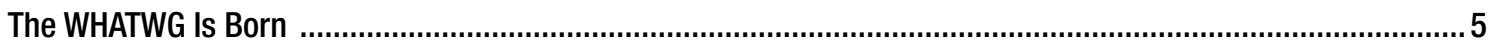

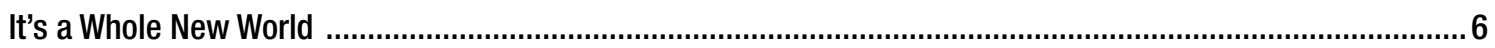

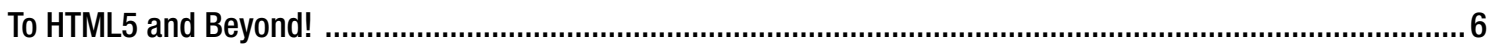

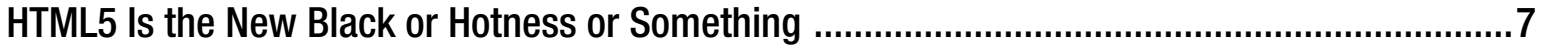

Is HTML5 Hype, Substance, or Both? ...............................................................................................

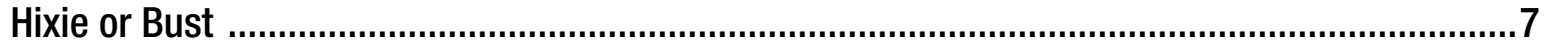

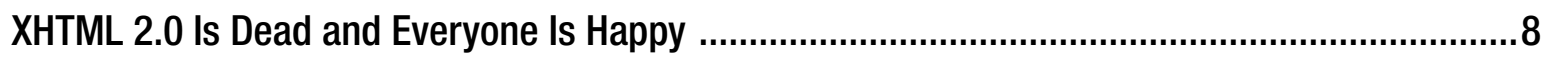

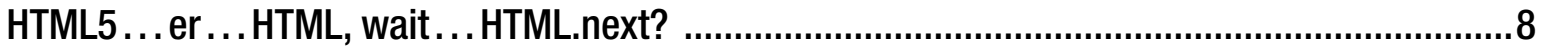




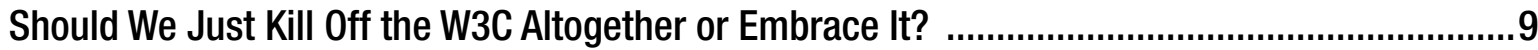

Reform

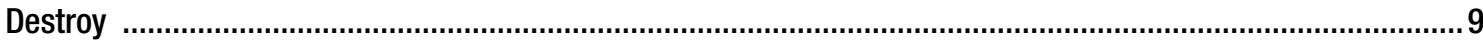

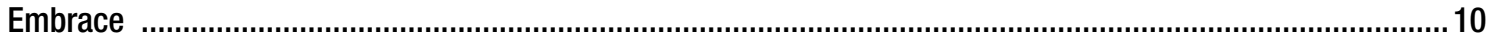

How Does New Stuff Get Added to HTML5 Now? ................................................................. 10

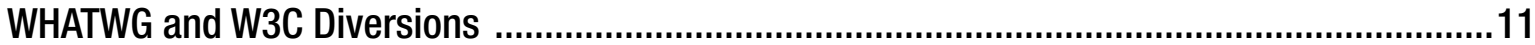

TL;DR

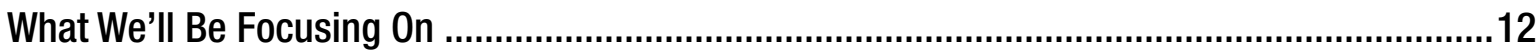

Chapter 2: The Truth About a Basic HTML5 Web Page.......................................................13

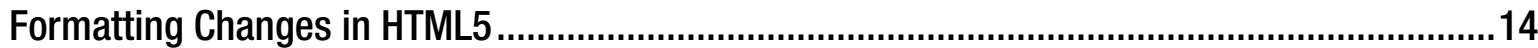

What About an HTML5 Shim and CSS for the New Elements? ...............................................14

What About the HTML5 Boilerplate and Modernizr?...............................................................15

Chapter 3: The Truth About Structuring an HTML5 Page ................................................17

A Little Taste of Pain: The Sectioning Elements......................................................................17

Where Did These Elements Come From? ..............................................................................18

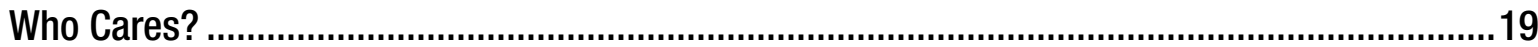

The Contradiction at the Heart of HTML5's New Elements ...................................................19

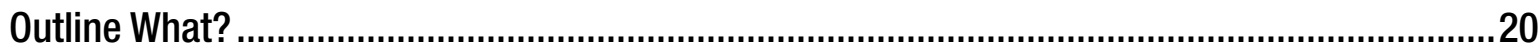

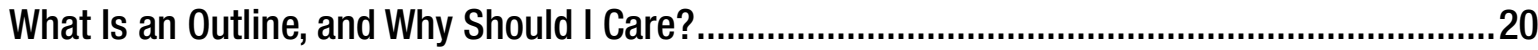

How We Currently Create Outlines (Even Without Realizing It) ...............................................20

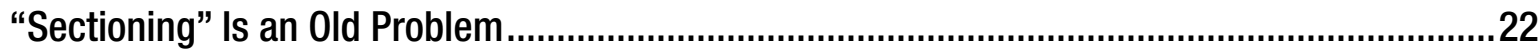

If We Care About Blind Users, We Should Care About Headings...............................................23

HTML5's “Improved" Outlining Was Dead Before It Ever Shipped...............................................23

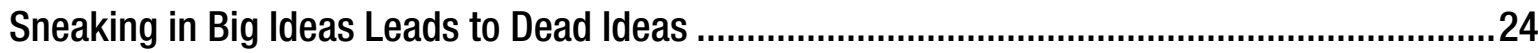

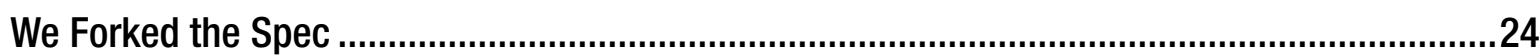

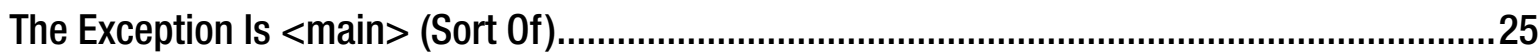

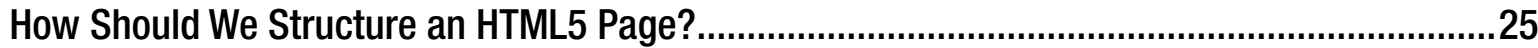

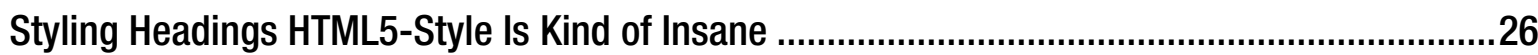


This Is Not Inconsequential: People Have to Teach This Stuff ................................................27

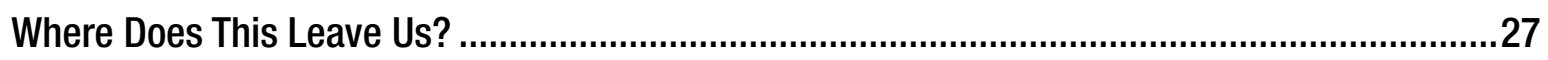

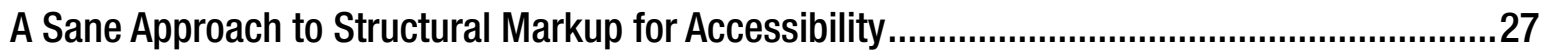

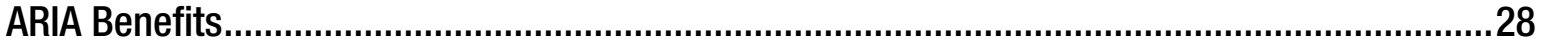

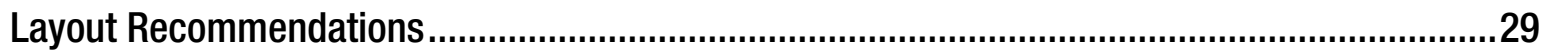

Chapter 4: The Truth About HTML5's Structural Elements............................................31

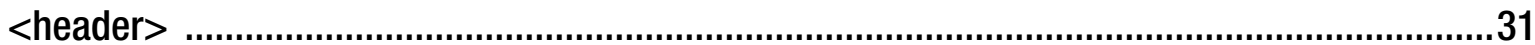

Really, It Doesn't Do Anything ....................................................................................................... 32

How Can Screen Readers Use <header > When It's Everywhere? .................................................................. 32

ARIA Alternative: Banner ................................................................................................................. 32

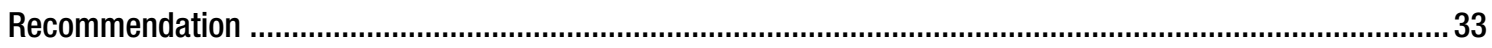

$<$ nav>

Good Intentions; Accessibility Disaster ................................................................................................. 33

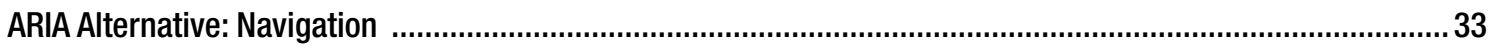

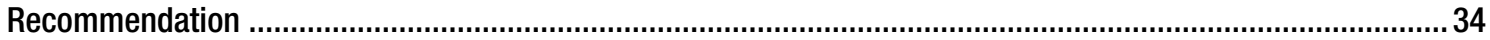

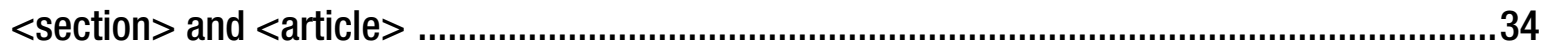

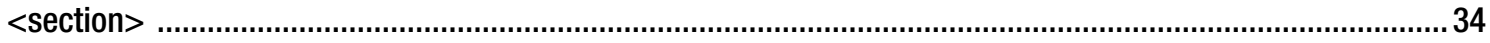

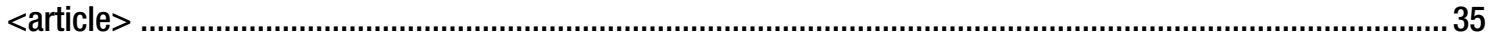

So, What's the Difference Between <article > and <section>? ....................................................................... 37

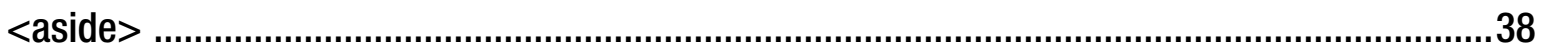

An Aside Creates an Outline Section in Weird Places ........................................................................ 38

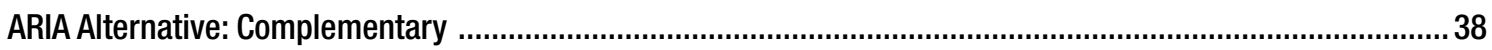

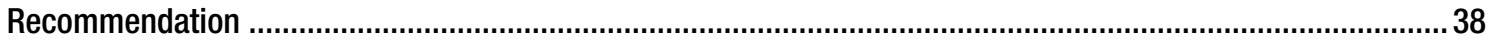

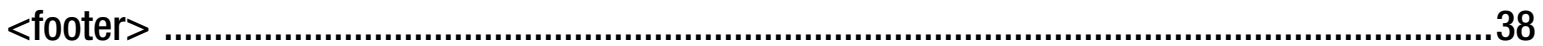

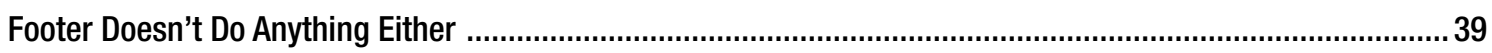

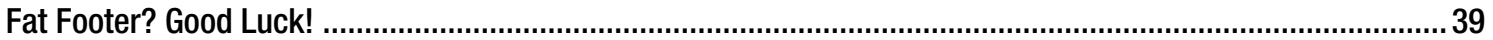

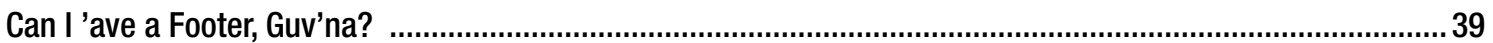

ARIA Alternative: contentinfo .......................................................................................................... 39

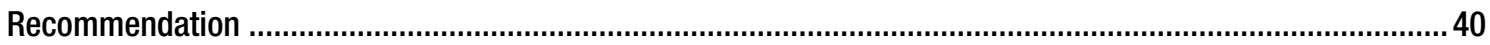


$<$ main $>$

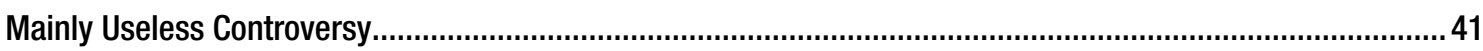

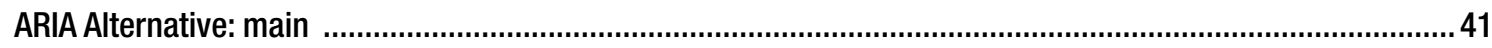

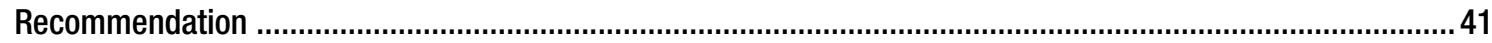

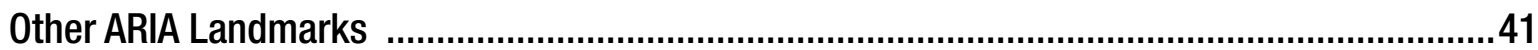

A Funny Thing Happened...Graceful Degradation Died and JavaScript Became Mandatory .....42

Yahoo's JavaScript Research ................................................................................................................... 42

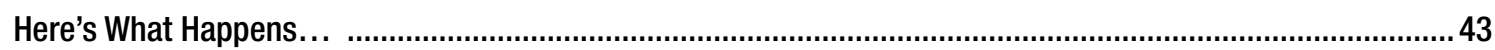

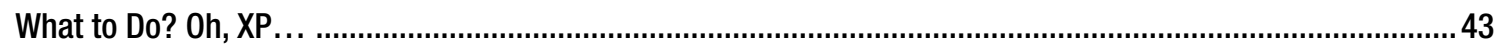

Uh...Web Design Community, What Happened? .................................................................4 44

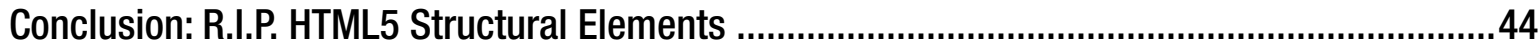

Chapter 5: The Truth About HTML5 Micro-semantics and Schema.org ..........................47

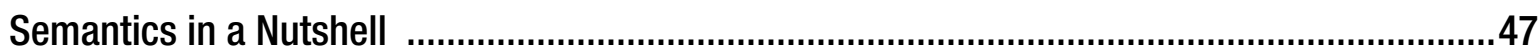

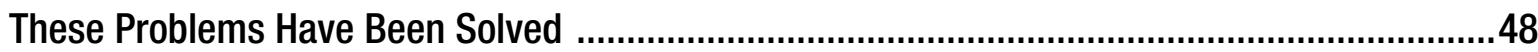

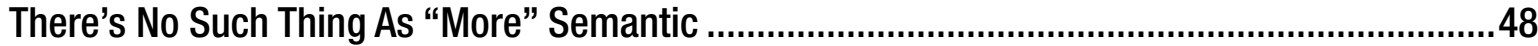

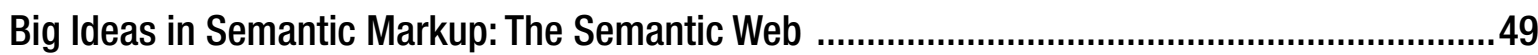

Semantics: Not Dead Yet (Or: Google \& Co Drop a Micro-Semantic Bombshell) ........................49

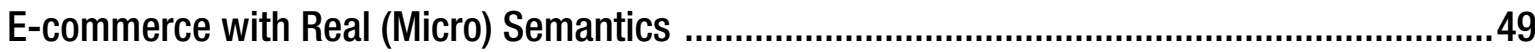

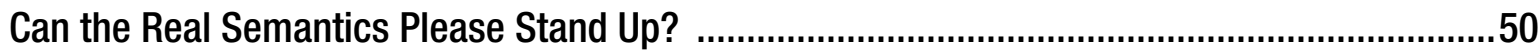

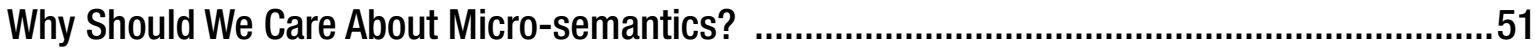

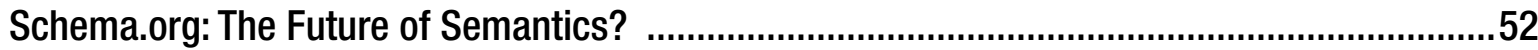

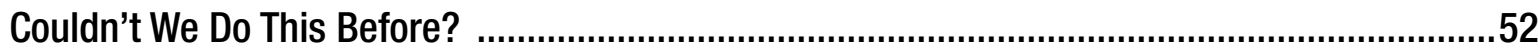

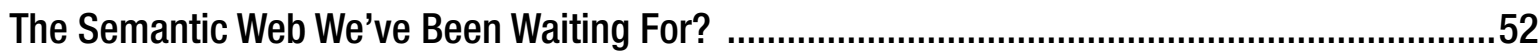

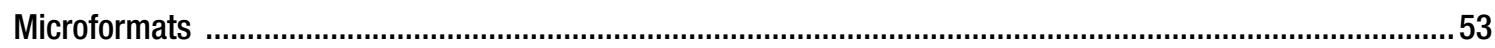

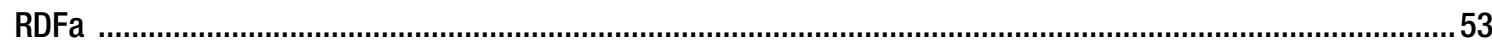

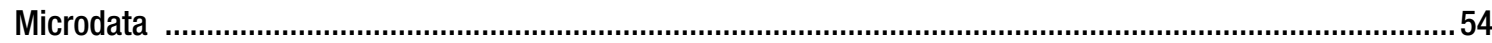

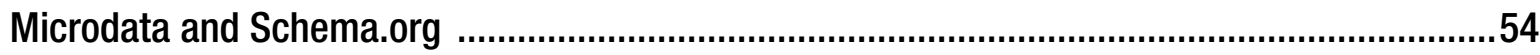

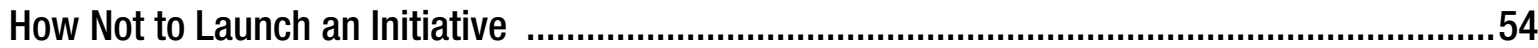

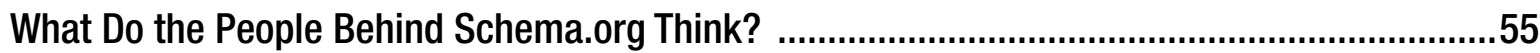

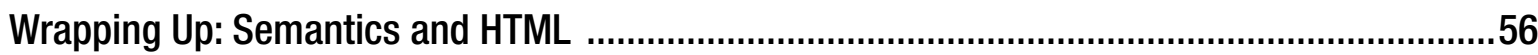


Chapter 6: The Truth About HTML5 and SEO

SEO in the Dark Ages

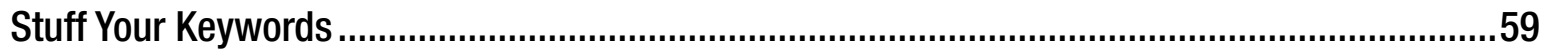

HTML and SEO

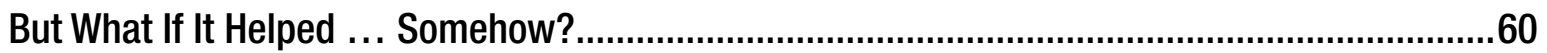

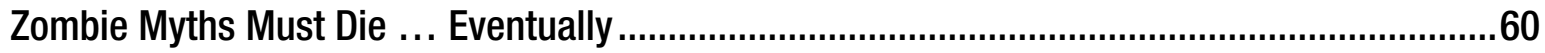

Chapter 7: The Truth About HTML5's Other New Elements ..............................................61

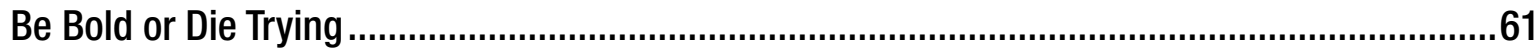

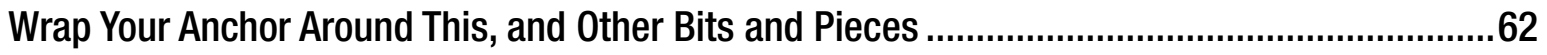

Wrap Anchors Around Block-Level Elements............................................................................................ 62

$<$ mark>

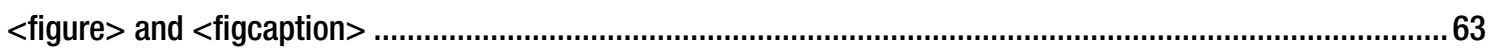

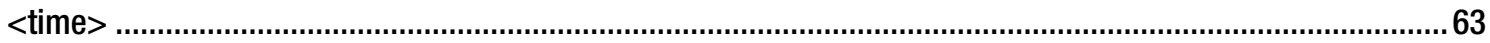

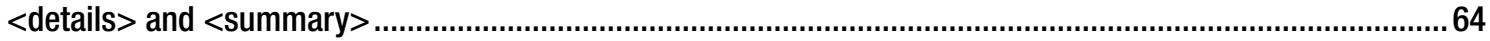

$<$ small>

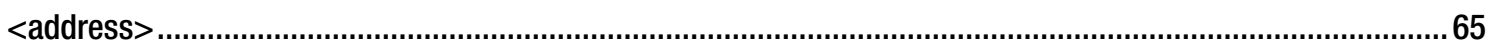

$<$ cite>

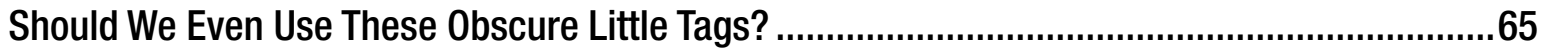

Chapter 8: The Truth About HTML5 Forms.......................................................................67

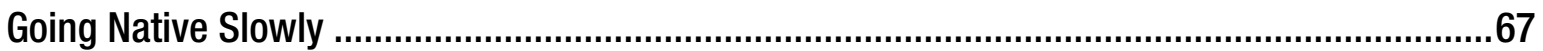

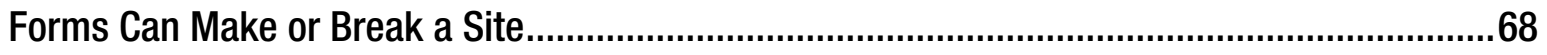

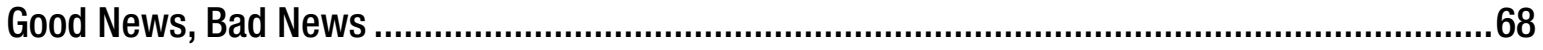

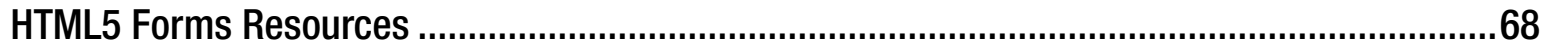

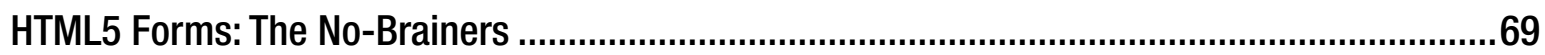

New Input Types: E-mail, URL, Telephone Number, and Search......................................................................69

Attributes: Autocomplete, Autofocus, Readonly, and Spellcheck ..................................................................70

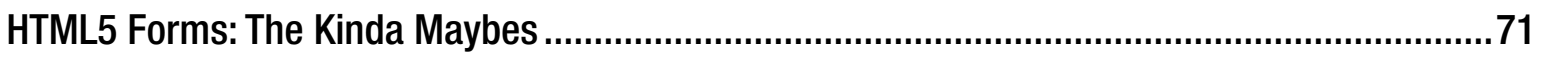

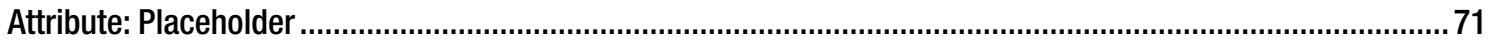

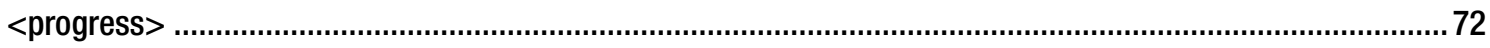

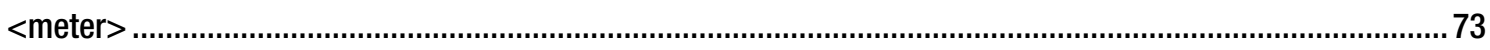


HTML5 Forms: The "I Wouldn’t Yet But You Can If You Really Want” ............................................74

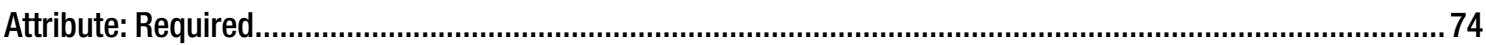

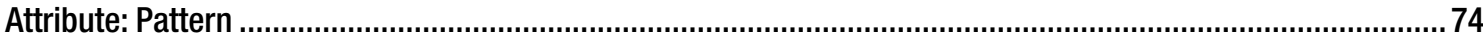

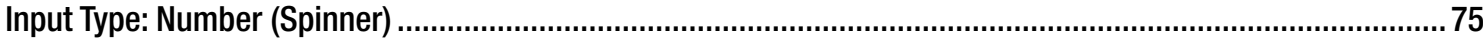

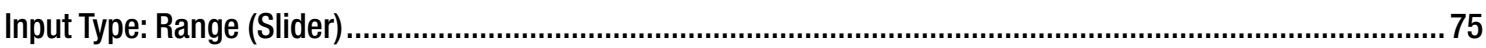

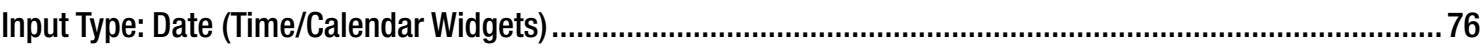

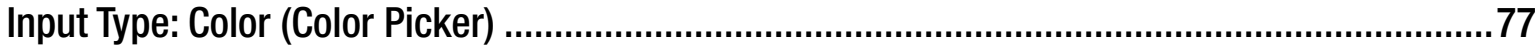

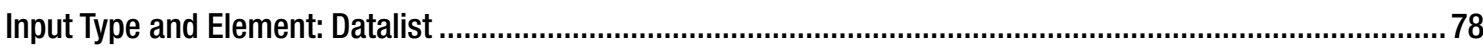

You Hypocrite. I Thought Requiring JavaScript Was the Worst Thing Ever ................................78

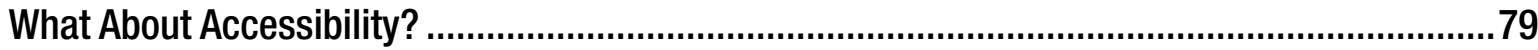

Chapter 9: The Truth About HTML5's Canvas, Gaming, and Flash......................................81

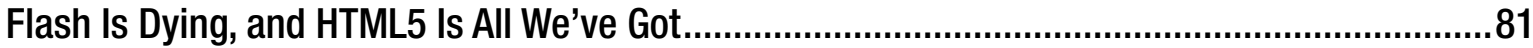

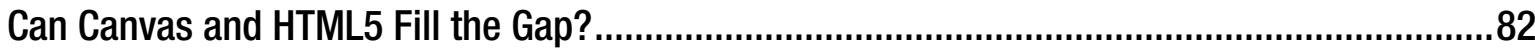

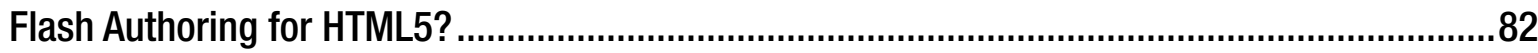

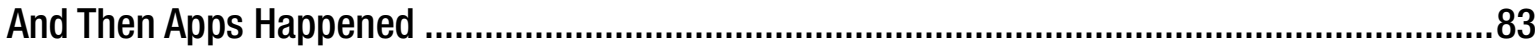

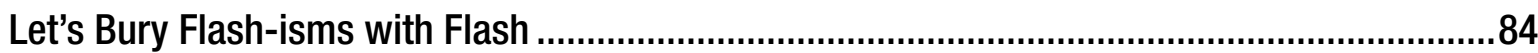

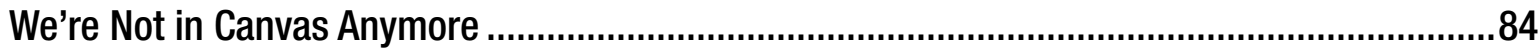

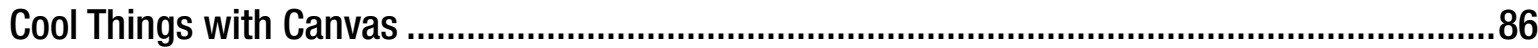

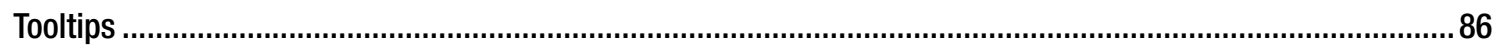

Charts

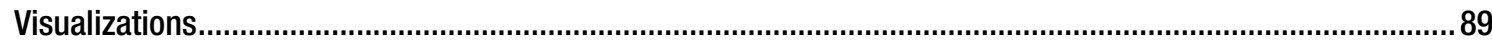

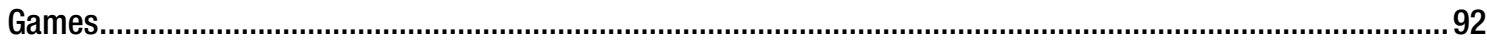

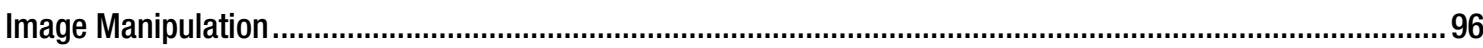

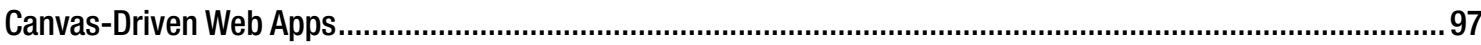

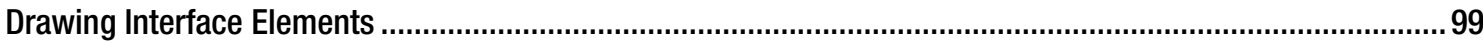

The Sometimes Good and Sometimes Bad Canvas Emulation for IE6-8....................................................... 102

The Haphazard World of Web Standards (Or: How Did We End Up with Canvas?).....................103

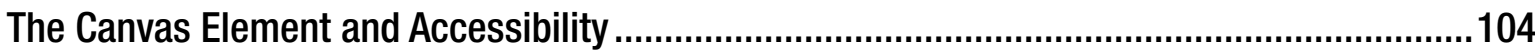




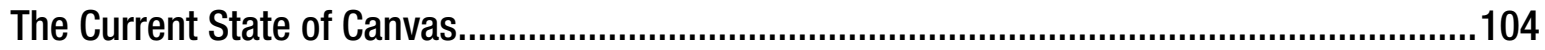

Primitive Development Environment ................................................................................................... 104

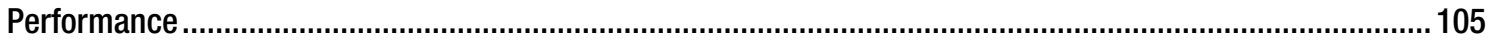

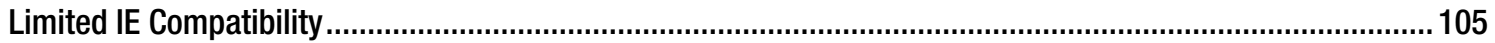

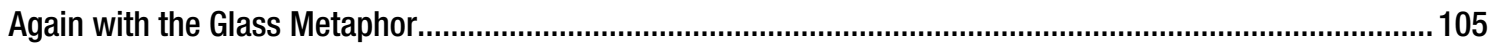

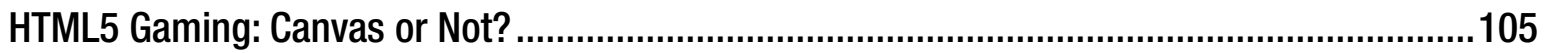

Is It Even Canvas?

Getting Started with Canvas Game Development.............................................................106

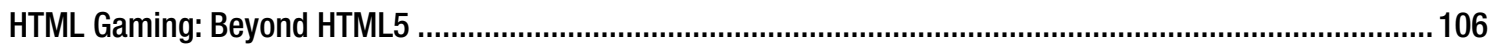

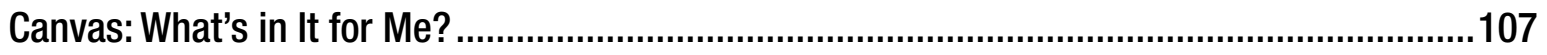

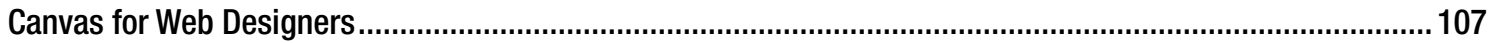

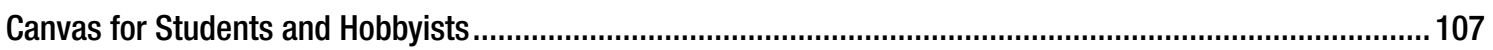

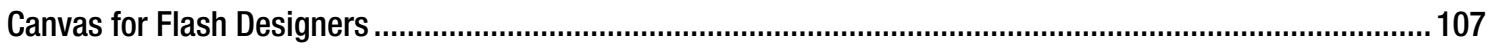

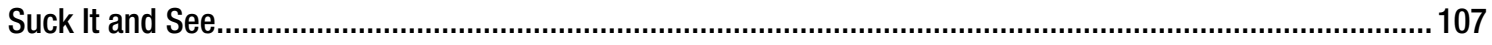

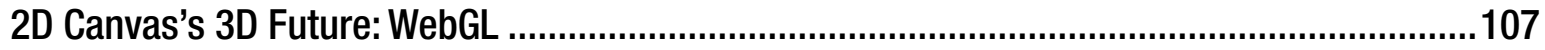

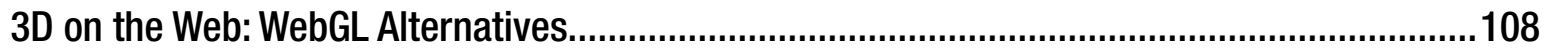

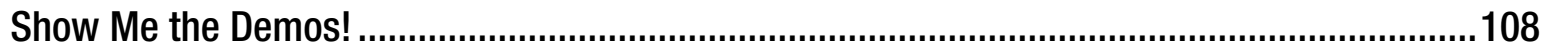

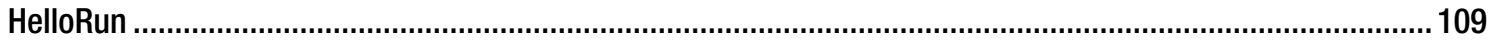

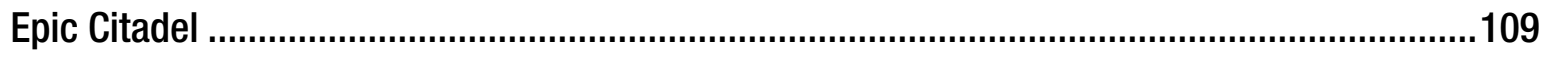

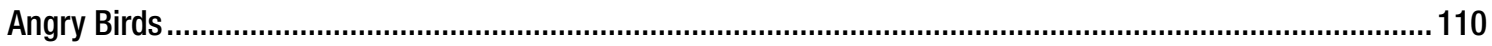

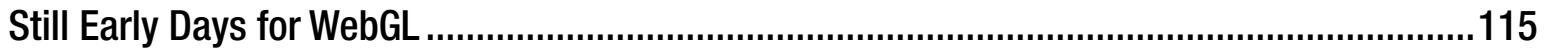

Chapter 10: The Truth About Audio and Video in HTML5 .......................................117

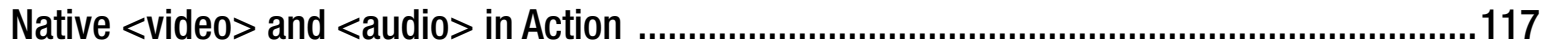

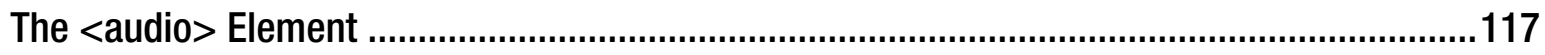

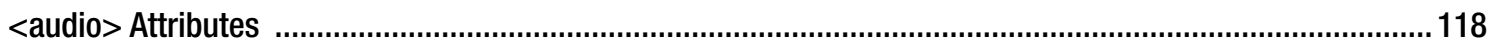

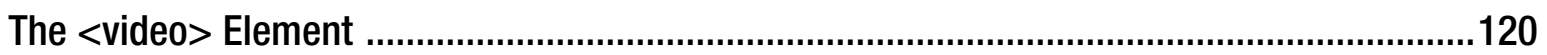

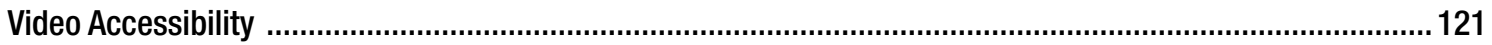

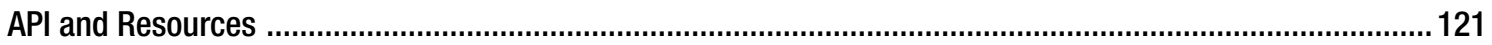

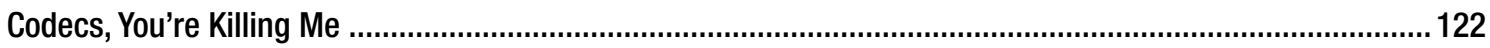




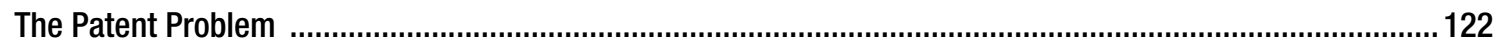

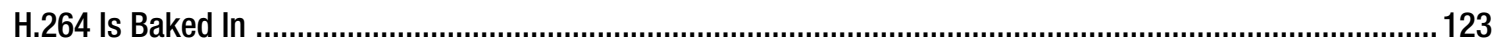

Google Threatens to Take Chrome WebM Only....and Then Doesn't ........................................................... 123

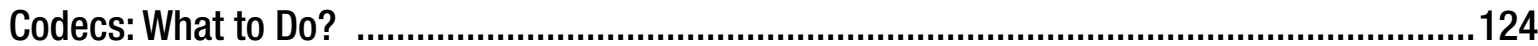

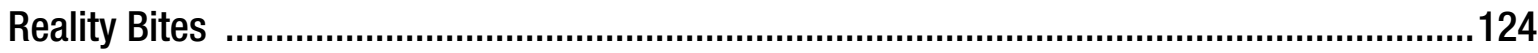

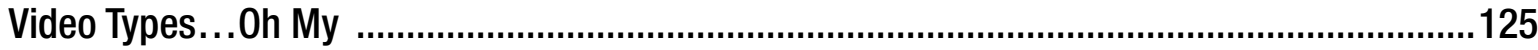

Querying Supported Video Types with JavaScript ...............................................................125

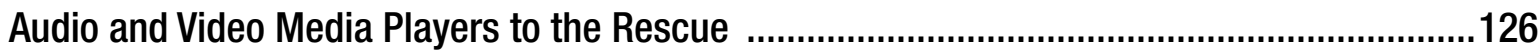

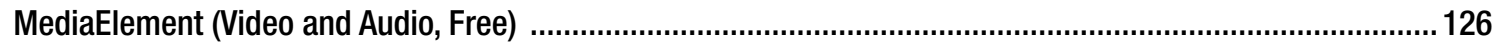

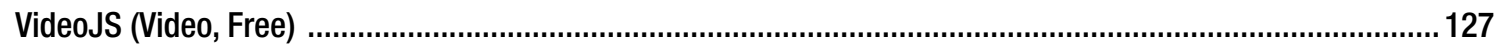

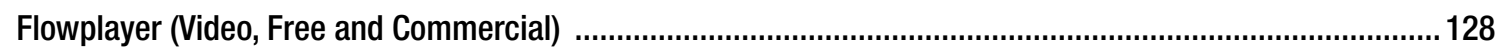

More Media Players .................................................................................................................... 129

0ther Flies in the HTML5 Video Ointment: DRM, Streaming, and Full-Screen Video ................130

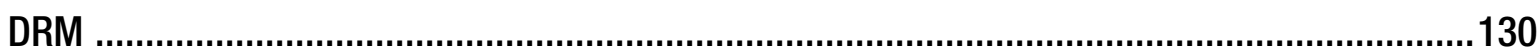

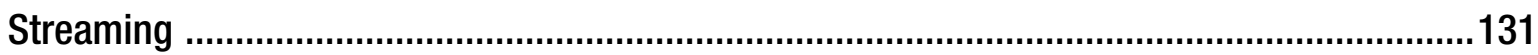

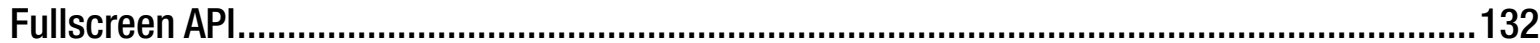

Is HTML5 <audio > Ready for Games? ........................................................................... 132

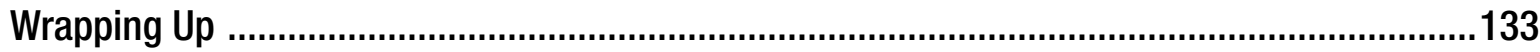

Chapter 11: The Truth About SVG: The Flash Challenger That Was,

Wasn't, and Now... .......................................................................135

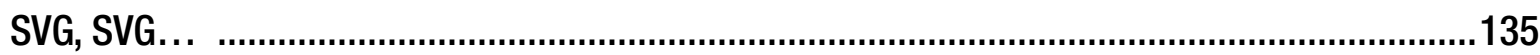

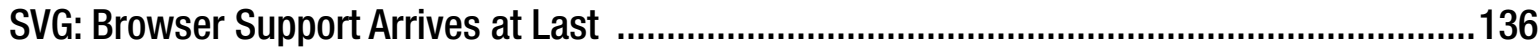

Yes, There Is Real-World SVG We Can Use Right Now ........................................................137

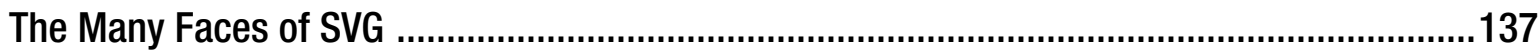

SVG in the '00s: The Great Hope That Wasn't ....................................................................137

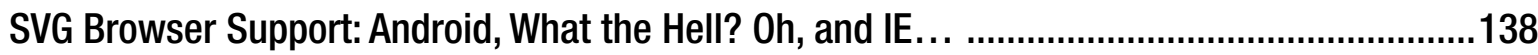

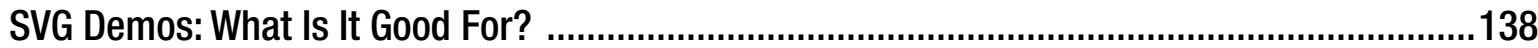

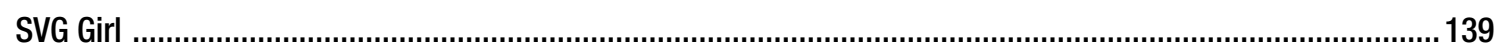

D3.js

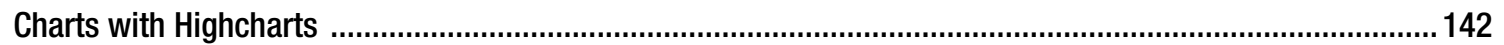




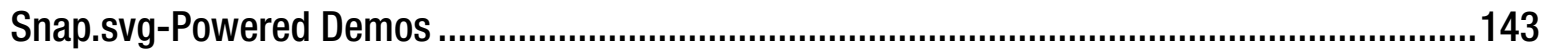

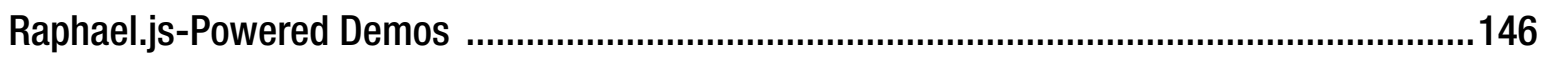

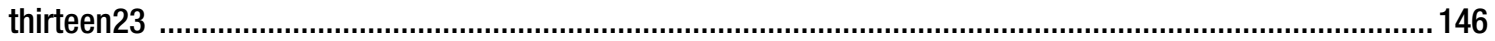

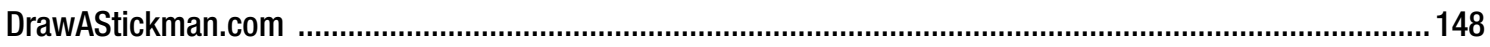

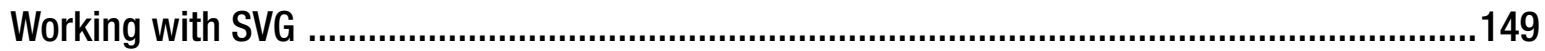

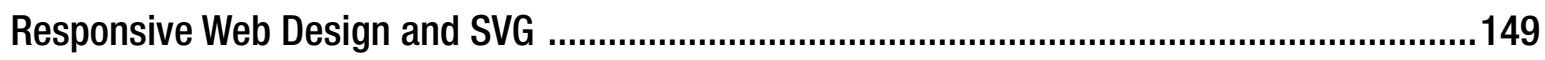

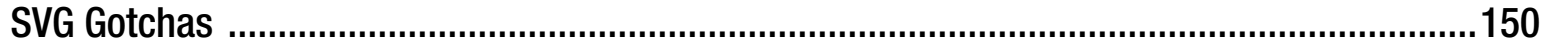

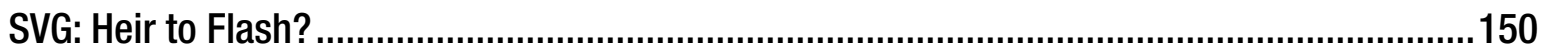

Chapter 12: The Truth About HTML5 Web Apps, Mobile, and What Comes Next............153

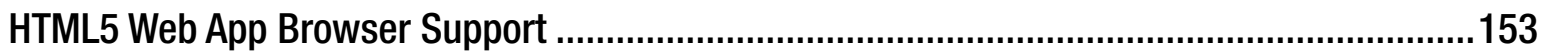

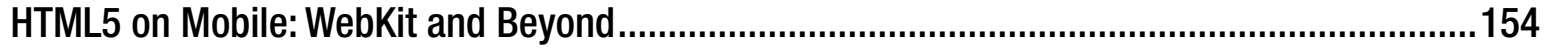

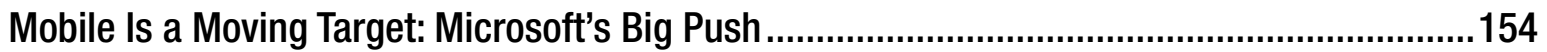

Firefox OS: Mozilla's Ambitious Mobile Platform, and the WebAPI ...........................................155

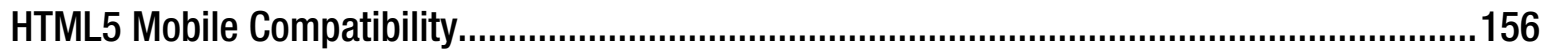

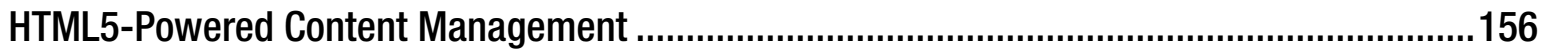

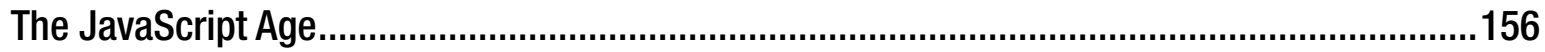

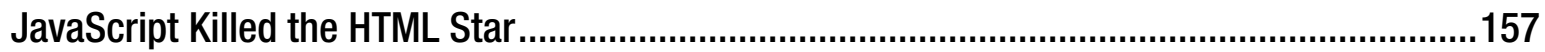

Modernizr, When Can I Use ... , and Polyfills ......................................................................158

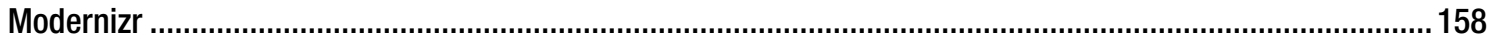

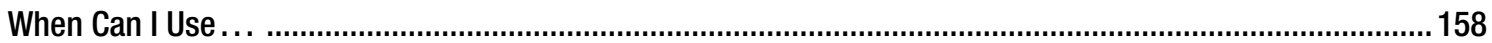

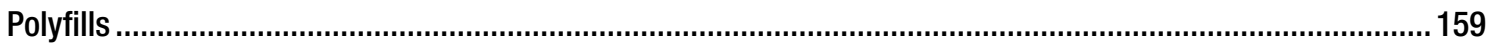

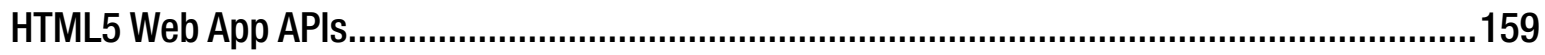

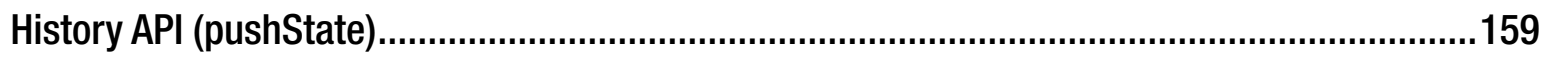

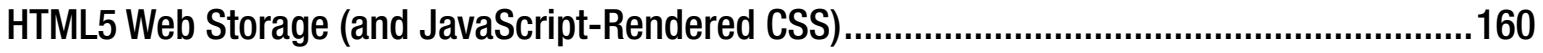

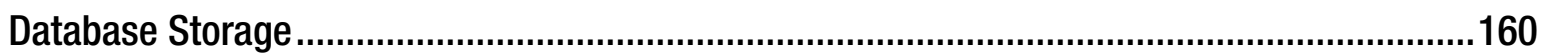

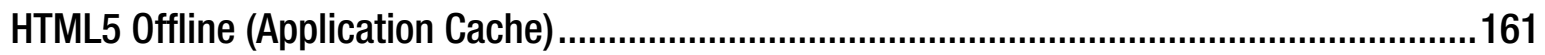

Geolocation API 


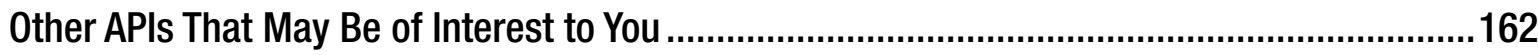

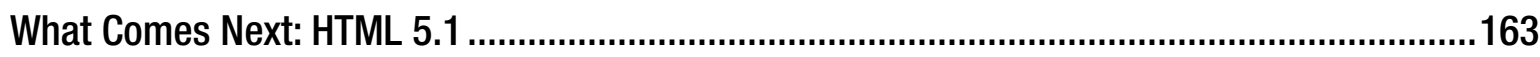

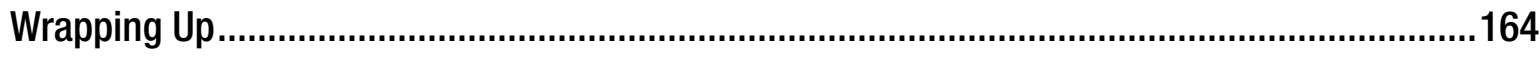

Chapter 13: The Truth About the Future of Web Design: Performance-Based Design......165

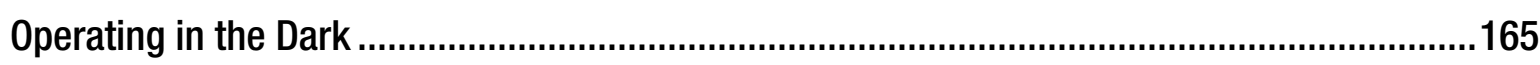

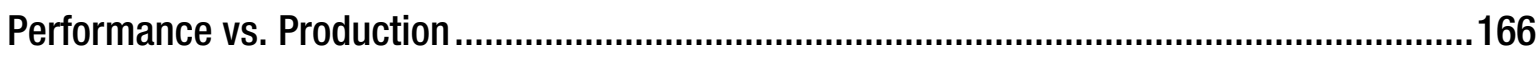

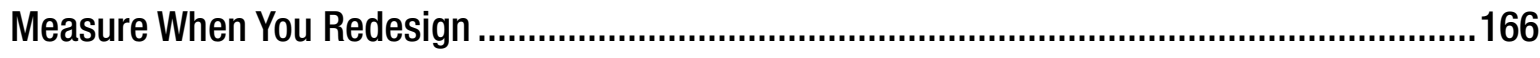

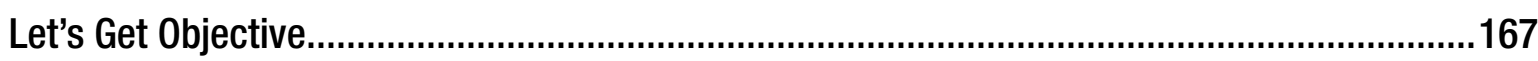

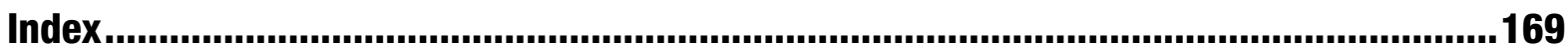




\section{About the Authors}

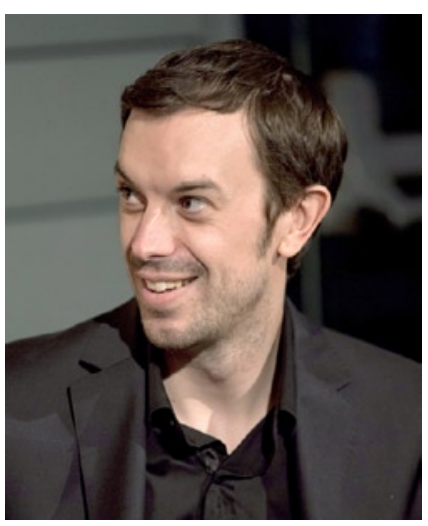

Luke Stevens is a Sydney-based designer who cut his teeth with PageMill in the 90s, wrangled Content Management Systems in the 00s, and switched to web apps in the 10s. With a passion for design, some brief formal print design training, and more than a decade of insatiable curiosity about new web technology, he has built sites of all shapes and sizes for clients all over the world, usually with ExpressionEngine. Currently in-between client work he tweets nonsense @lukestevens and works on his web app at AnalyticsExplorer . com which he will, he swears, release in 2014.

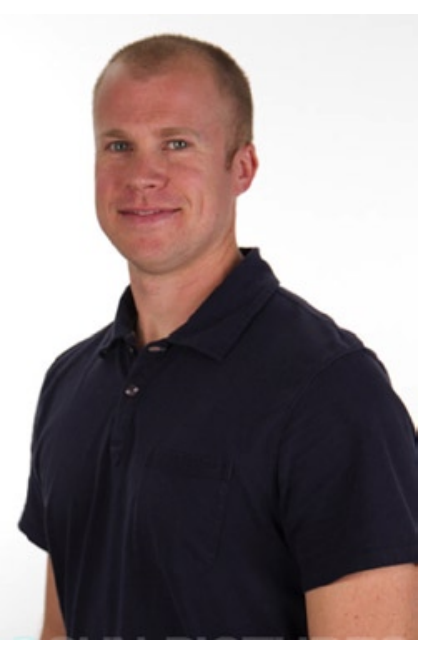

RJ Owen is an experienced front-end developer who has worked in a variety of technologies on the Web for more than ten years. He is a frequent speaker at industry events such as SXSW and Adobe's Max conference. RJ started his career as a Flash developer before moving to the open Web and then to design and customer insight work, which he totally loves. When not working or writing, RJ spends time chasing his children or enjoying the mountains of Colorado, where he lives and works. 



\section{About the Technical Reviewer}

Andrew Zack has been at the forefront of implementing successful Internet marketing strategies since 1996. He has authored a book and been a technical reviewer for numerous industry publications. Mr. Zack's industry expertise includes business development, IT project management, Internet marketing, SEO implementation, operational software, and web site development and coordination. 



\section{Foreword}

HTML5 is a mess. It's also one of the most exciting technological advances perhaps ever (a big claim, especially for something I just described as a mess).

There are quite a few books, most of them excellent, on HTML5. Some cover the markup exclusively. Some cover markup and JavaScript APIs. Others still focus on a specific development challenge, such as games.

This book is a little different. Rather than simply looking at the what and how of HTML5 (though it does that as well), it endeavors to explain the why and why not of HTML5.

And it's a passionate, informed, opinionated critique of much of HTML5 to boot.

Along the way, you'll learn a great deal about HTML5 markup and additional HTML5 features such as the new audio and video elements, the Canvas element, the History API, and related features such as SVG.

But ideally, most of all you'll learn to think critically about HTML5 as a tool and adopt the good parts, for good reasons, and ignore the less than useful parts, for the right reasons as well.

Luke Stevens has written a book all web designers and developers who care about their code should read. So go ahead and read it!

John Allsopp

Author, Developing with Web Standards

Cofounder of Web Directions

Web evangelist 



\section{Introduction}

\section{Introduction From RJ}

Hi. I'm RJ, your average HTML developer. I've been working with code since I was a kid and quickly gravitated to the instant gratification that comes from making beautiful things for computers, especially online.

I did the updates and revisions for the most recent version of this book, and I have to tell you, you are in for a wild ride. A year ago I was just like you-a person who hadn't read this book. I came into it thinking HTML5 was pretty neat. I had done a few "HTML5" sites, read a few blog posts, enjoyed the fancy logo, and maybe even bought the official HTML5 T-shirt (yeah, I did). I liked the new elements because they were, you know, semantic. I liked evolving beyond Flash. I liked (the idea of) SVG.

Wow, did I have a lot to learn. What follows from here is a journey into a strange and unbelievable world. If you're like I was, then very little of what comes next is going to be what you expect, and even less of it will seem reasonable. HTML5 is not what you think it is. Buckle up: Luke Stevens is taking us all to school, and between you and me he drives a little like a maniac.

\section{Introduction From Luke}

Hi. I'm Luke, your average, garden-variety web designer. I've been building web sites for more than a decade, use ExpressionEngine as my CMS, and have enjoyed both working in-house and full-time freelancing.

I thought it would be fun to write a short book about HTML5. I thought HTML5 would be simple. I thought writing about it would be straightforward. And I thought the respected voices in the design community would be telling everyone what it is (and what it isn't) simply and clearly, particularly with the plethora of other HTML5 books out there.

I was wrong.

Fortunately, this book (and I hope your experience as a reader!) is infinitely better for it. And I hope once you've read it you'll share my concern about the strange direction basic markup has taken and my excitement for the new HTML5 (and related) technologies that are coming soon to a browser near you. That includes Internet Explorer - Microsoft, finally, truly gets web standards.

What seemed impossible just a few years ago-a far-fetched, almost utopian ideal of all browser vendors, including Microsoft, competing tooth-and-nail to support bleeding-edge web standards-is now a reality. Innovation in web standards is happening at break-neck speed, and my hope is this book gets you up to speed not only with the fundamentals of HTML5 but with the broader picture of where the Web as a whole is heading, especially as we look toward a post-Flash future.

As you make your way through the following chapters, please keep in mind this book is as much of a critique as it is an explanation of HTML5. By taking a critical look at why things are the way they are, my hope is you save hours by not having to worry about things that don't matter (particularly when it comes to basic markup) and your eyes are opened to how the HTML5 sausage gets made. It may not always be pretty, but if you spend your days in the trenches building web sites, knowing why things are the way they are will help guide your design and development decisions in a very direct way. 
That said, there's plenty of exciting technology in and around HTML5 too, so be sure not to miss the later chapters on graphics technologies like Canvas and SVG, the state of audio and video in HTML5, and the more developeroriented HTML5 features including a new way of handling something as fundamental as a page request.

(Also note we will be focusing almost entirely on HTML5 as defined by the HTML5 spec, with the addition of SVG and a few other related initiatives such as Schema.org and WebGL. "HTML5" has become a buzzword that can mean everything from the HTML5 spec itself to CSS3 and modern JavaScript to just "cool and new and not Flash." We'll be mostly sticking with the features in the actual HTML5 specification.)

I love the web design community because it's filled with smart, excitable, curious, opinionated folk who will call you on your BS. This is an opinionated book, not a dry explanation of the technology, and I'll be stating my views pretty strongly. I look forward to you doing the same. Passionate, considered debate makes us all smarter.

So please, write it up on your blog, send me happy/sad/angry e-mails (luke@itsninja.com), talk to me on Twitter (@ lukestevens), or whatever you like.

I look forward to the discussion.

And now I'd like to ask a couple of favors.

First, if you enjoy my writing, then please tell your friends, colleagues, Twitter followers, blog readers, and pretty much anyone who will listen about this book. Like a lot of authors, I rely on readers like you to spread the word (and the links). If you can help me out by spreading the word about this book via good old-fashioned word of mouth, I'd really appreciate it. Thank you.

And second, if you use Google Analytics and want to get more out of it, I'd love you to check out my web app Analytics Explorer (http: //analyticsexplorer .com). Analytics Explorer brings all the great data that Google Analytics buries to the surface through a simple, elegant interface, and combines it with great tips on improving your site. You can add your clients, send them nicely designed regular reports, and focus your work on improving metrics that matter, not just making the proverbial logo bigger. My hope is it will make your own design practice (and your client's sites) more productive and profitable. After all, all the HTML5 in the world won't help you if your conversion rates are lousy and your bounce rates are sky-high. (We'll return to this theme in the final chapter of this book when we look at Performance Based Design.) Check it out: http: //analyticsexplorer.com. 